\title{
Fission of metallic clusters in the liquid drop model
}

\author{
Armando Vieira, Carlos Fiolhais \\ Departamento de Física, Universidade de Coimbra, 3000 Coimbra, Portugal
}

Received 5 March 1996; revised manuscript received 30 May 1996; accepted for publication 20 June 1996

Communicated by B. Fricke

\begin{abstract}
A.bstract
Using the liquid drop model and the jellium model, we calculate fission barrier heights as a function of charge and mass asymmetry for a family of shapes consisting of two spheres connected by a quadratic surface. We find the fissibility for which a mass asymmetric splitting gives place to the symmetric one (Bussinaro-Gallone point) and evaluate the size of charged clusters of alkali metals for which the fission barrier height is equal to the evaporation energy (critical sizes). The results for the critical sizes agree very well with experiment.
\end{abstract}

PACS: $36.40 . \mathrm{Qv} ; 36.40 . \mathrm{Wa}$

\section{Introduction}

We know from experiment that metallic atomic clusters with excess charge are unstable due to the tnagnitude of their Coulomb energy. These clusters undergo two processes: breaking out in charged pieces (fission) or expelling a neutral atom (evaporation). Evaporation is preferred for large clusters, but fission prevails for small clusters. For a critical cluster size, $N_{\mathrm{c}}$, where fission barriers and evapora tion energies are comparable, the two decay processes compete [1].

From the theoretical point of view, the liquid drop model (LDM) combined with the jellium model [2] (where the ions are replaced by a continuous positive background) is a useful tool to explain cluster fission. In a charged cluster two oppositc forces cxist. The surface tension obliges the system to have a
Iniminal surface. On the other side, the electrostatic repulsive force due to the extra charge drives to expansion and deformation. Fission occurs when the second force surpasses the first. This can only happen if the energy of the fragments is lower than the energy of the mother cluster, i.e., when the reaction heat (or $Q$-value) is negative. However, since the process is controlled by a barrier, knowledge of the barrier height is essential to evaluate the decay probability associated to each channel.

Fission of metallic clusters displays remarkable similarities with nuclear fission where the LDM is well known. But metallic clusters show an additional degree of freedom with respect to nuclei, namely that we are, in principle, free to consider any charge in the parent and daughter clusters. Based on the LDM, we will evaluate, for different charged metal clusters fission barriers along different fission channels. We 
compute the Coulomb energy exactly, for a given family of shapes [3], assuming that the charge is bound to the surface. Based on the barrier heights, we analyze the asymmetry in mass distribution between the fragments. The critical numbers $N_{\mathrm{c}}$ are also estimated for multiply charged clusters of some alkali metals.

Cluster fission has been studied by several authors. Bréchignac et al. [1] measured the critical size for doubly charged clusters of sodium and potassium, and were also able to determine the fission barrier for some doubly charged clusters [4]. Näher et al. [5,6] obtained the critical size of alkali metal clusters with up to seven electrons removed.

On the theoretical side, Saunders [7] used the LDM to predict some critical sizes for doubly charged clusters of sodium and gold. He calculated $N_{c}=30$ for $\mathrm{Na}$ and $N_{\mathrm{c}}=18$ for Au using the LDM, but considering only symmetric reactions. Näher et al. [6] applied the LDM, with the so-called "funny hills" parametrization for the cluster shapes (including asymmetric configurations), but did not predict the observed critical numbers. Garcias et al. [8] used a semi-empirical model for the Coulomb interaction between the emergent fragments, obtaining a good description of the critical numbers. Similar results were achieved by Fröbrich [9], employing an empirical model for that interaction and using fission decay rates. Recently, Vieira and Fiolhais [10] and Yannouleas and Landman [11] have used the two-centre shell model to obtain shell corrections to the LDM fission barriers of small clusters. There are also a few molecular dynamics calculations of fission [12].

The IDM is introduced in Section 2, where we compare reaction heats with fission barriers heights as a function of mass and charge asymmetry and evaluate the Bussinaro-Gallone point, i.e., the fissibility corresponding to the instability of the barrier against mass asymmetry. Section 3 contains the results on critical sizes, and conclusions.

\section{Liquid drop model and fission barriers}

In the framework of the LDM, the energy of a spherical metallic cluster with $N$ monovalent atoms and $z$ lacking electrons (the charge is $q=+e z$ ), is given by

$$
\begin{aligned}
E_{\mathrm{LDM}}(N, z)= & a_{v} V+\sigma S+\gamma C \\
& +z\left(W+\frac{c}{R_{0}}\right)+\frac{(e z)^{2}}{2\left(R_{0}+d_{s}\right)},
\end{aligned}
$$

where $V=\frac{4}{3} \pi R_{0}^{3}$ is the volume, $S=4 \pi R_{0}^{2}$ is the surface area, $C=2 \pi R_{0}$ is half the mean curvature, and $R_{0}=r_{s} N^{1 / 3}$ is the radius of the cluster, $r_{s}$ being the density parameter. The parameters $a_{v}, \sigma, \gamma$ are the liquid drop coefficients for a neutral cluster. The term with $c$ is a first order quantal correction to the work function $W$ related to the finite size of the cluster. The last term is the classical electrostatic energy, obtained under the assumption that the cluster is a perfect conductor. The parameter $d_{s}$ accounts for the charge spill-out effect: the excess charge lies on a radial centroid displaced $d_{s}$ from the jellium surface. The volume term will not be considered since volume is conserved during deformation and fragmentation.

The LDM is known to provide a good average of quantal density functional results for the reaction heats [13]. Let us consider the binary fission of a spherical cluster with $N$ atoms and charge $z$ in two spherical pieces with $N_{1}$ and $N_{2}=N-N_{1}$ atoms and charges $z_{1}$ and $z_{2}=z-z_{1}$, respectively. For simplicity and convenience, we set, for the time being, $\gamma=c-d_{s}=0$. The energy released, divided by the surface energy $E_{\mathrm{S}}^{0}$, may be written as

$$
\begin{aligned}
& Q(x, \alpha, \beta)=\left[E_{\mathrm{LDM}}\left(N_{1}, z_{1}\right)+E_{\mathrm{LDM}}\left(N_{2}, z_{2}\right)\right. \\
& \left.-E_{\mathrm{LDM}}(N, z)\right] / E_{\mathrm{S}}^{0} \\
& =\left[\alpha^{2 / 3}+(1-\alpha)^{2 / 3}-1\right] \\
& +2 x\left(-\frac{\beta^{2}}{\alpha^{1 / 3}} \frac{(1-\beta)^{2}}{(1-\alpha)^{1 / 3}} \quad 1\right) \text {, }
\end{aligned}
$$

where $x=(e z)^{2} / 16 \pi \sigma r_{s}^{3} N$ is the quotient between the Coulomb and twice the surface energy, called the fissibility parameter. For $x=1$, the initial system is unstable relatively to fission, while, for $x=0$, the 
system is neutral and cannot fragment. The parameters $\alpha=N_{1} / N$ and $\beta=z_{1} / z$ measure the asymmetry of mass and charge of the fragments. Eq. (2) loolds in nuclear physics with $\beta=\alpha$ [14].

Fig. 1a shows $Q(x=0.5, \alpha, \beta)$. The minimum of $Q$ is located at $(\alpha=0.07, \beta=0.32)$. If $x$ increases, this minimum moves up along the bold line, $3_{\min }=\Delta(\alpha)$, with

$d(\alpha)=\frac{\alpha^{1 / 3}-(1-\alpha)^{1 / 3}}{\alpha^{1 / 3}+(1-\alpha)^{1 / 3}}$.

Reactions with high $\beta$ and low $\alpha$ and vice versa are aighly disfavorable.

A negative reaction heat is only a necessary condition for a cluster to break. In order to estimate decay probabilities of charged clusters we need to evaluate barriers heights for each channel. In particular, we should look for the channels with the lowest energy barriers.

To know the barrier height we need to extend the LDM formula to deformed shapes, corresponding to intermediate fission configurations. The surface term in Eq. (1) is then replaced by $E_{\mathrm{S}}=\sigma \int \mathrm{d} A$, while the electrostatic energy, $E_{\mathrm{C}}$, is calculated numerically assuming, as before, that all excess charge is distributed on the surface. We solve the Poisson equation following the method described in Ref. [15].

To parametrize the cluster surface we adopt Blocki's shapes, which consists of two spheres joined by a smooth neck $[3,16]$. This family of shapes is described by three parameters measuring elongation, neck width, and mass asymmetry, defined respectively by:

$\rho=m \frac{d}{R_{1}+R_{2}}, \quad \lambda=\frac{l_{1}+l_{2}}{R_{1}+R_{2}}, \quad \Delta=\frac{R_{1}-R_{2}}{R_{1}+R_{2}}$,

where $d$ is the center to center distance, $R_{1}=$ $R_{0} \alpha^{1 / 3}$ and $R_{2}=R_{0}(1-\alpha)^{1 / 3}$ are the radii of the two fragments, and $l_{1}$ and $l_{2}$ are the thickness of the lens-shaped piece of a sphere which lies within the matching quadratic surface. The parameter $\Delta$ is the same as in Eq. (3).

If we define $B_{\mathrm{S}}=E_{\mathrm{S}} / E_{\mathrm{S}}^{0}$ and $B_{\mathrm{C}}=E_{\mathrm{C}} / E_{\mathrm{C}}^{0}$, the LDM energy for a deformed cluster, $E_{\mathrm{LDM}}=$ $E_{\mathrm{LDM}}(N, q, \rho, \lambda, \Delta)$, after convenient normalization, is given by

$\frac{E_{\mathrm{LDM}}}{E_{\mathrm{S}}^{0}}=B_{\mathrm{S}}+2 x B_{\mathrm{C}}$.

The normalized energy barrier becomes

$\xi=\frac{E_{\mathrm{LDM}}^{\mathrm{sad}}-E_{\mathrm{LDM}}^{\mathrm{sph}}}{E_{S}^{0}}=\left(B_{\mathrm{S}}^{\mathrm{sad}}-1\right)+2 x\left(B_{\mathrm{C}}^{\mathrm{sad}}-1\right)$,

where $E_{\mathrm{LDM}}^{\mathrm{sad}}$ and $E_{\mathrm{LDM}}^{\mathrm{sph}}$ are the energies of the saddle and the spherical shapes, and $B_{\mathrm{S}}^{\text {sad }}$ and $B_{\mathrm{C}}^{\text {sad }}$
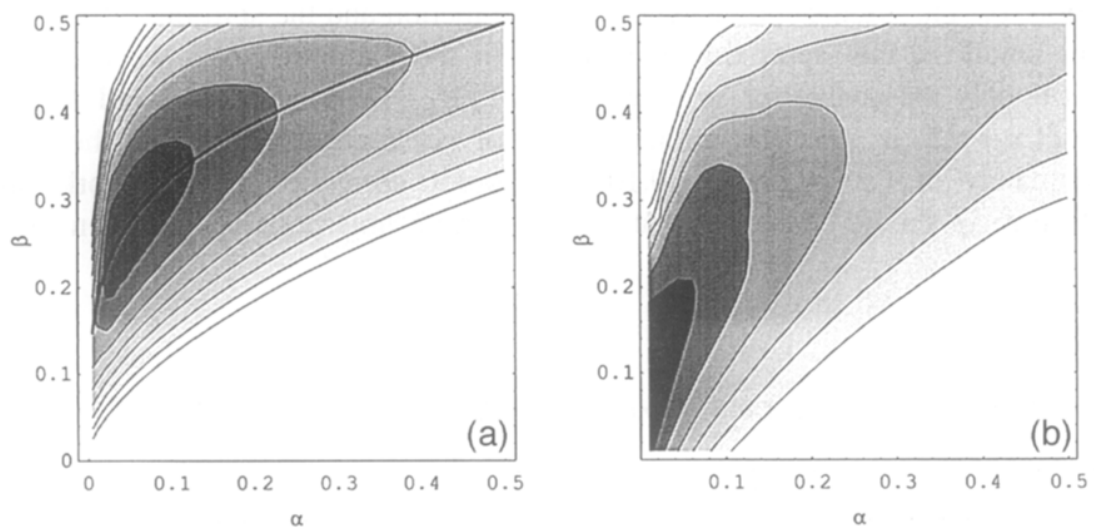

Fig. 1. (a) $Q$-values $Q(x=0.5, \alpha, \beta)$ (only negative values are represented) as a function of mass and charge asymmetry (see text for the definition of $\alpha$ and $\beta$ ). The bold line represents $\beta_{\min }=\Delta(\alpha)$ (see Eq. (3)). (b) Barrier height divided by the surface energy of the initial spherical cluster, $\xi(x-0.5, \alpha, \beta)$, as a function of the sanne variables. 


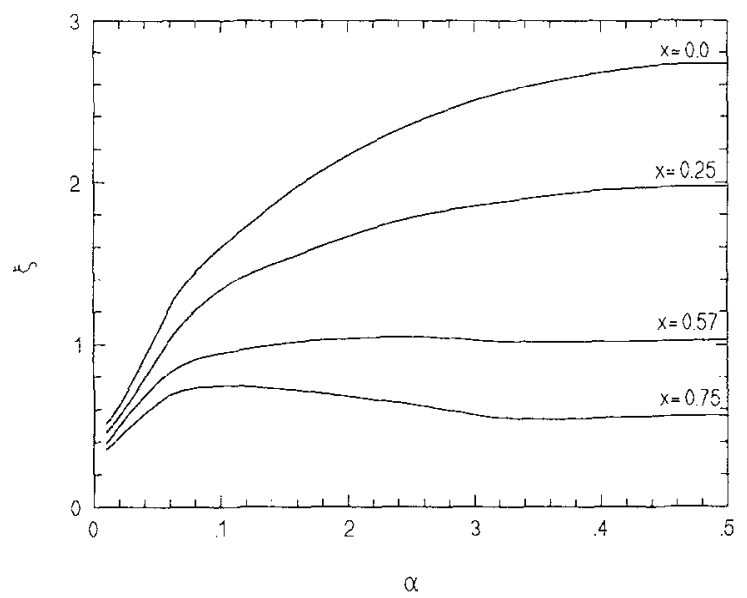

Fig. 2. Dimensionless barrier height, $\xi(x, \alpha, \beta=\alpha)$, for several values of the fissibility $x$. The Bussinaro-Gallone fissibility, $x_{\mathrm{BG}}$, occurs when the second derivative of $\xi$ with respect to $\alpha$, at $\alpha=0.5$, changes sign. We found $x_{\mathrm{BG}}=0.57$.

are $B_{\mathrm{S}}$ and $B_{\mathrm{C}}$ evaluated at the saddle point in the variables $\rho, \lambda$ ( $\Delta$ is fixed).

To search for the saddle point, we started by evaluating the minimum of the energy along the fission line defined by the equation $\lambda=1-1 / \rho$. We then proceeded backwards following the line of fast variation of the neck $[10,16], \lambda=1-\sqrt{\rho-k}$, with $k$ a constant, up to $\lambda=1$. From there, we have followed the path $\lambda=1-\Delta^{2} / \rho$. For small $x$, the saddle point is located close to the fission line. As $x$ approaches 1 , the saddle point moves to configurations closer to a sphere. Since we have assumed a path, our evaluation of the saddle point is only approximative. For small $x$, this approximation is very good, while it is only reasonable for large $x$.

Fig. 1b shows $\xi(x=0.5, \alpha, \beta)$. The minimum is located approximately at $(\alpha=0.01, \beta=0.10)$. The similarity with Fig. la should be pointed out.
In Fig. 2 we present $\xi(x, \alpha, \beta=\alpha)$. As in nuclear fission, the asymmetric reaction is preferred for small $x$ and the symmetric reaction is preferred for large $x$. However, the transition from asymmetric to symmetric fission, the so-called Bussinaro-Gallone point, occurs at $x_{\mathrm{BG}}=0.57$, where for nuclei $x_{\mathrm{BG}} \simeq$ 0.40 , the precise value depending on the particular shape parametrization [17-19]. This disagreement should be due to the difference in the charge distribution.

\section{Critical numbers}

A very important number is the cluster size $N=N_{\mathrm{c}}$ for which the barrier height equals the evaporation energy. For each charge $z$, we have scarched for a solution of the equation

$E_{\mathrm{LDM}}^{\mathrm{sad}}(N, z, \alpha, \beta)-E_{\mathrm{LDM}}^{\mathrm{sph}}(N, z)=E_{\mathrm{eva}}(N, z)$,

where $E_{\mathrm{eva}}(N, z)=E_{\mathrm{LDM}}(N-1, z)+E_{\mathrm{LDM}}(1,0)$ $-E_{\mathrm{LDM}}(N, z)$ is the evaporation energy. We assume, as in Ref. [8], that the preferred fission channel is $[N]^{z+} \rightarrow[N-3]^{z-1+}+[3]^{+}$, i.e., $\alpha=3 / N$ and $\beta=1 / z$. This channel is the most common in the experimental data.

Since we have assumed an asymmetric channel, we consider now, for the sake of precision, the curvature energy, the size correction of the work function and the spill-out effect in the LDM formula. In the curvature term we use is $C=\frac{1}{4} \int\left(1 / R_{\min }+\right.$ $\left.1 / R_{\max }\right) \mathrm{d} A$, with $R_{\min }$ and $R_{\max }$ the principal radii of curvature at a point of the surface. For the first order correction to the work function we use a simple interpolation between the initial spherical

Table 1

LDM coefficients for Na, K and Cs. The values of $a_{s}=4 \pi \sigma r_{s}^{2}$ and $a_{\mathrm{c}}=2 \pi \gamma r_{s}$, were taken from Ref. [20], $c$ from Ref. [21], and $d_{s}$ from Ret. $[22]$

\begin{tabular}{llllll}
\hline Metal & $r_{s}(\mathrm{bohr})$ & $a_{s}(\mathrm{eV})$ & $a_{\mathrm{c}}(\mathrm{eV})$ & $c(\mathrm{eV})$ & $d_{s}$ (bohr) \\
\hline $\mathrm{Na}$ & 3.93 & 0.57 & 0.25 & -2.26 & 1.32 \\
$\mathrm{~K}$ & 4.96 & 0.54 & 0.17 & -2.26 & 1.17 \\
$\mathrm{Cs}$ & 5.62 & 0.50 & 0.09 & -2.26 & 1.02 \\
\hline
\end{tabular}


Table 2

LDM critical sizes, $N_{c}$, of charged clusters of $\mathrm{Na}, \mathrm{K}$ and $\mathrm{Cs}$, in comparison with experimental data and other theoretical results

\begin{tabular}{|c|c|c|c|c|c|c|c|}
\hline & & \multicolumn{6}{|l|}{$z$} \\
\hline & & 2 & 3 & 4 & 5 & 6 & 7 \\
\hline \multirow[t]{4}{*}{ Pa } & our work & 26 & 68 & 129 & 209 & 311 & 404 \\
\hline & Ref. [8] & 26 & 63 & 117 & 185 & 268 & 366 \\
\hline & Ref. [9] & 22 & 62 & 123 & 208 & 316 & 451 \\
\hline & exp. (Ref. [6]) & $27 \pm 1$ & $63 \pm$ & $123 \pm 2$ & $206 \pm 4$ & $310 \pm 10$ & $445 \pm 10$ \\
\hline \multirow[t]{3}{*}{$\mathrm{K}$} & our work & 23 & 58 & 110 & 176 & 242 & 344 \\
\hline & Ref. [8] & 24 & 59 & 110 & 173 & 249 & 337 \\
\hline & exp. (Ref. [6]) & $20 \pm 1$ & $55 \pm 1$ & $110 \pm 5$ & & & \\
\hline \multirow[t]{3}{*}{ Cs } & our work & 22 & 57 & 105 & 168 & 233 & 330 \\
\hline & Ref. [8] & 23 & 57 & 105 & 165 & 236 & 319 \\
\hline & $\exp ($ Ref. [6]) & $19 \pm 1$ & $49 \pm 1$ & $94 \pm 1$ & $155 \pm 2$ & $230 \pm 5$ & $325 \pm 10$ \\
\hline
\end{tabular}

cluster and final spherical fragments. The spill-out effect is taken into account by placing the charge on a surface which is separated by $d_{s}$ from the cluster surface. Table 1 shows the values of the LDM zoefficients used in our calculations.

Table 2 displays the critical numbers $N_{\mathrm{c}}$ obtained for several clusters of simple metals and compares them with experimental results. A very good agreement between our LDM theory and experiment is observed (for potassium we are not aware of data for $z>4$ ). We should remark that the LDM does not predict necessarily the channel $[3]^{+}$.

Included in Table 2 are the results of Garcias et al. [16] and Fröbrich [9]. The first authors have used

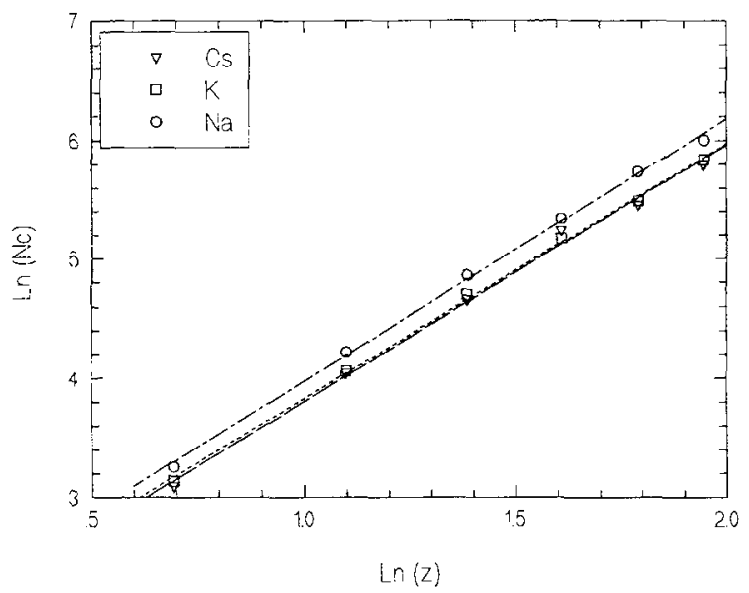

Fig. 3. Logarithm of the critical size, $\ln N_{c}$, as a function of the logarithm of the number of missing electrons, in $z$, for clusters of $\mathrm{Na}, \mathrm{K}$ and $\mathrm{Cs}$, determined in the framework of the LDM theory. A straight line fits the results very well. a different surface energy and have not included $R$ and $1 / R$ effects. They have fitted the Coulomb energy to Thomas-Fermi results in a somewhat ad hoc fashion. On the other hand, the comparison of the fission and evaporation decay rates as done by Fröbrich [9] is, in principle, a better method for estimating $N_{\mathrm{c}}$ than the rule given by Eq. (7). (In that method the $Q$-values are also taken into account.) But we stress that the Coulomb energy is computed exactly in our work, while in Ref. [9] it is fitted to reproduce experimental results. Moreover this author considers the fragmentation channel $[N]^{z+} \rightarrow$ $[N-1]^{z-1+}+[1]^{+}$which is unlikely to occur in practice since it has a higher $Q$ than the channel we take.

If we plot the critical sizes in a logarithmic scale, the points lie approximately on a straight line, $\ln \left(N_{\mathrm{c}}\right)$ $=s \ln (z)+$ const (Fig. 3). We find $s=2.20$ for $\mathrm{Na}$, $s=2.15$ for $\mathrm{K}$ and $s=2.16$ for Cs. These slopes are lower than the average experimental value $2.3 \mathrm{ob}-$ tained in Ref. [6]. They are higher than the value 2 proposed by Rayleigh on the simple assumption that a charged drop breaks when the electrostatic energy equals the surface energy.

In the present work, we have shown how the mass distribution and the fission threshold are evaluated in the LDM. In a pure LDM analysis shell effects are absent. The role of shell fluctuations in the barrier height should be investigated systematically. However, we expect the LDM to have a reasonable predictive power for critical numbers of large enough clusters, for which shell corrections are less important. 


\section{Acknowledgement}

This work has been partially supported by the Commission of the European Communities under contract No. ERB4002PL-910205 and by the Praxis XXI project No. 2/2.1/FIS/26/94. One of us (A.V.) has been supported by a grant of the Praxis XXI program.

\section{References}

[1] C. Bréchignac, Ph. Cahuzac, F. Carlier and M. Frutos, Phys. Rev. Lett. 64 (1990) 2893.

[2] M. Brack, Rev. Mod. Phys. 65 (1993) 677.

[3] J. Blocki, J. Phys. (Paris) 45 C 6 (1984) 489.

[4] C. Bréchignac, Ph. Cahuzac, F. Carlier and M. de Frutos, Phys. Rev. B 49 (1994) 2825;

C. Bréchignac, Ph. Cahuzac, F. Carlier, M. de Frutos, P. Garnier and N. Kebali, Phys. Rev. B 53 (1996) 1091.

[5] U. Näher, H. Goehlich, T. Lange and T.P. Martin, Phys. Rev. B 68 (1992) 3416.
[6] U. Näher, S. Frank, N. Malinowski, U. Zimmermann and T.P. Martin, Z. Phys. D 31 (1994) 191.

[7] W.A. Saunders, Phys. Rev. B 46 (1992) 7028.

[8] F. Garcias, R.J. Lombard, M. Barranco, J.A. Alonso and J.M. López, Z. Phys. D 33 (1995) 301.

[9] P. Fröbrich, Phys. Lett. A 202 (1995) 99.

[10] A. Vieira and C. Fiolhais, Z. Phys. D, in press.

[11] C. Yannouleas and U. Landman, in: Large clusters of atoms and molecules, Proc. NATO ASI, Eric, June 19-22 (1995), in press.

[12] R.N. Bamett, U. Landman and G. Rajagopal, Phys. Rev. Lett. 67 (1991) 3058.

[13] A. Vieira, M. Brajczewska and C. Fiolhais, Int. J. Quant. Chem. 56 (1995) 239.

[14] L. Wilets, Theories of nuclear fission (Oxford Univ. Press, Oxford, 1964).

[15] H. Koizumi, S. Sugano and Y. Ishii, Z. Phys. D 28 (1993) 223.

[16] F. Garcias, A. Mañanes, J.M. López, J.A. Alonso and M. Barranco, Phys. Rev. B 51 (1995) 1897.

[17] U.L. Bussinaro and S. Gallone, Nuovo Cim. 1 (1955) 629.

[18] R. Hasse, Ann. Phys. (NY) 68 (1971) 377.

[19] J.R. Nix, Nucl. Phys. A 130 (1969) 241.

[20] C. Fiolhais and J.P. Perdew, Phys. Rev. B 45 (1992) 6207.

[21] M. Seidl and M. Brack, Ann. Phys. (NY) 245 (1995) 275.

[22] A. Kiejna, Phys. Rev. B 47 (1993) 7361. 